\title{
How Much Do We Know about Maintaining Treatment Response after Successful Acne Therapy? Systematic Review on the Efficacy and Safety of Acne Maintenance Therapy
}

\author{
Corinna Dressler Stefanie Rosumeck Alexander Nast
}

Division of Evidence-Based Medicine (dEBM), Department of Dermatology, Charité - Universitätsmedizin Berlin, Berlin, Germany

\section{Key Words}

Acne vulgaris - Maintenance - Systematic review .

Recurrence $\cdot$ Adapalene Benzoyl peroxide $\cdot$ Tazarotene . Isotretinoin

\begin{abstract}
After cessation of successful initial acne therapy, patients often experience flares. Consecutive maintenance treatment after successful induction therapy is promoted by guidelines; however, little is known about the efficacy/safety of different maintenance regimens. A systematic review on acne maintenance treatments was conducted. We identified 5 randomized controlled trials [RCTs; adapalene vs. vehicle or vs. no treatment (3 RCTs), adapalene/benzoyl peroxide (BPO) vs. vehicle, combination/monotherapy of minocycline (systemic)/tazarotene/placebo] and 3 non-RCTs on systemic isotretinoin, adapalene/BPO and azelaic acid. The results of adapalene versus vehicle/no treatment varied depending on the reported outcome. The 'number of patients maintaining at least 50\% improvement' counting inflammatory lesions/non-inflammatory lesions with adapalene was superior to vehicle (risk ratio, RR 1.24, 95\% confidence interval, $\mathrm{Cl}$ 1.08-1.43/RR 1.34, 95\% Cl 1.18-1.59). However, no significant differences were found in 2 of 3 RCTs for maintaining
\end{abstract}

'clear/almost clear' or 'mild acne' or on the global grading score. For the combination regimens of minocycline/tazarotene/placebo, no significant differences were found. Adapalene/BPO was superior to vehicle counting inflammatory lesions/non-inflammatory lesions (RR 1.61,95\% Cl 1.31-1.99; RR 1.80, 95\% Cl 1.44-2.26). Due to the scarcity of studies, few conclusions can be drawn. More homogeneous outcome measures and specific maintenance study designs may lead to more robust findings.

(c) 2016 S. Karger AG, Basel

\section{Introduction}

Acne is a very frequent skin disease, which, depending on its severity, can have a severe impact on the patients' quality of life. In contrast to the common perception of acne being a temporary pre-adolescents' and mostly cosmetic problem, in many patients acne persists for several years. It may progress to acne tarda, which is present in up to $40 \%$ of patients [1]. Even after resolution of the inflammatory active acne period, many patients are left with a significant amount of scarring, which affects selfesteem and can cause additional psychological distress later on [2].

\section{KARGER}

E-Mail karger@karger.com

www.karger.com/drm (c) 2016 S. Karger AG, Base

$1018-8665 / 16 / 2323-0371 \$ 39.50 / 0$ 


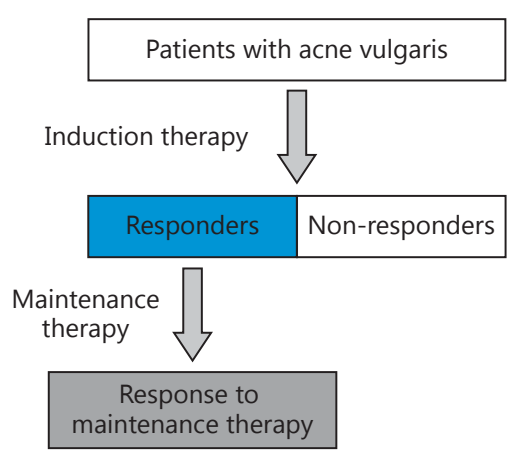

Fig. 1. Definition of responder population for maintenance therapy.

Table 1. Inclusion criteria

\begin{tabular}{|c|c|}
\hline PICOS & Description \\
\hline Population & $\begin{array}{l}\text { Adolescents and adults } \\
\text { Inclusion of patients who achieved a clearly } \\
\text { defined treatment goal (e.g. reduction in lesion } \\
\text { count by } 60 \% \text { ) before entering into the reported } \\
\text { maintenance study }\end{array}$ \\
\hline Intervention & $\begin{array}{l}\text { Approved systemic and topical interventions for } \\
\text { acne (other than anti-androgens and laser and } \\
\text { light therapies) }\end{array}$ \\
\hline Comparison & $\begin{array}{l}\text { With another drug and/or placebo, or without } \\
\text { comparison }\end{array}$ \\
\hline Outcome & Efficacy and safety/tolerability outcomes \\
\hline Study design & $\begin{array}{l}\text { Randomized controlled trial or controlled } \\
\text { interventional study with a minimum duration of } \\
12 \text { weeks; at least } 10 \text { participants per study arm }\end{array}$ \\
\hline
\end{tabular}

Most of the available studies have focused on induction therapy, and currently available systematic reviews have evaluated efficacy, safety and onset of action of antiacne treatments. Guidelines include maintenance therapy but only based on an 'expert opinion approach'. Thorough systematic review methods have yet to be utilized to inform these decisions.

In contrast to its relevance in daily practice, only few attempts have been made to identify optimal mainte- nance regimens. Questions such as how to best maintain treatment response after successful systemic isotretinoin or antibiotics therapy or how to continue on an initially successful but strongly irritating topical therapy with a better tolerable maintenance treatment need to be answered.

This systematic review summarizes the current evidence with regard to acne maintenance therapy. We aimed to:

1 provide a concise overview of published studies,

2 evaluate the quality of current studies, and

3 if suitable, combine outcome measures in meta-analyses.

\section{Methods}

For further details, see the supplementary materials (for all online suppl. material, see www.karger.com/doi/10.1159/000446069) [3-5] (fig. 1; table 1).

\section{Results}

Our initial search found 436 records. After removing duplicates, 280 records were left. By scanning reference lists another 4 and by auto-alerts (last search July 15, 2015) another 17 records were identified (fig. 2). Titles and abstracts of 301 records were screened for inclusion/ exclusion; 264 publications did not meet the predefined inclusion criteria. Twenty-seven full-text studies were excluded due to: no definition/evaluation of 'responder' (14), no original data (7), no relevant outcome data (2), no maintenance treatment (2), full text not obtainable (1), and less than 10 patients (1). We included 8 studies (data published in 10 different publications) reporting on adapalene gel $0.1 \%$, adapalene $0.1 \%$ /benzoyl peroxide (BPO) $2.5 \%$ fixed combination, tazarotene gel $0.1 \%$ and minocycline $100 \mathrm{mg}$ as well as azelaic acid (AA) $20 \%$.

Five studies were randomized controlled trials (RCTs), which will be described first. A summary of the 3 nonrandomized studies and data on relapse, adverse events and risk of bias will be presented for all included studies thereafter.

\section{Randomized Controlled Trials}

Five RCTs including a total of 983 participants (from 36 to 127 ) with a mean age range of 17.8-19.1 years and $43-64 \%$ female participants at baseline of the maintenance phase were included (table 2). The skin type/ethnic 
Fig. 2. Flow chart of record selection.

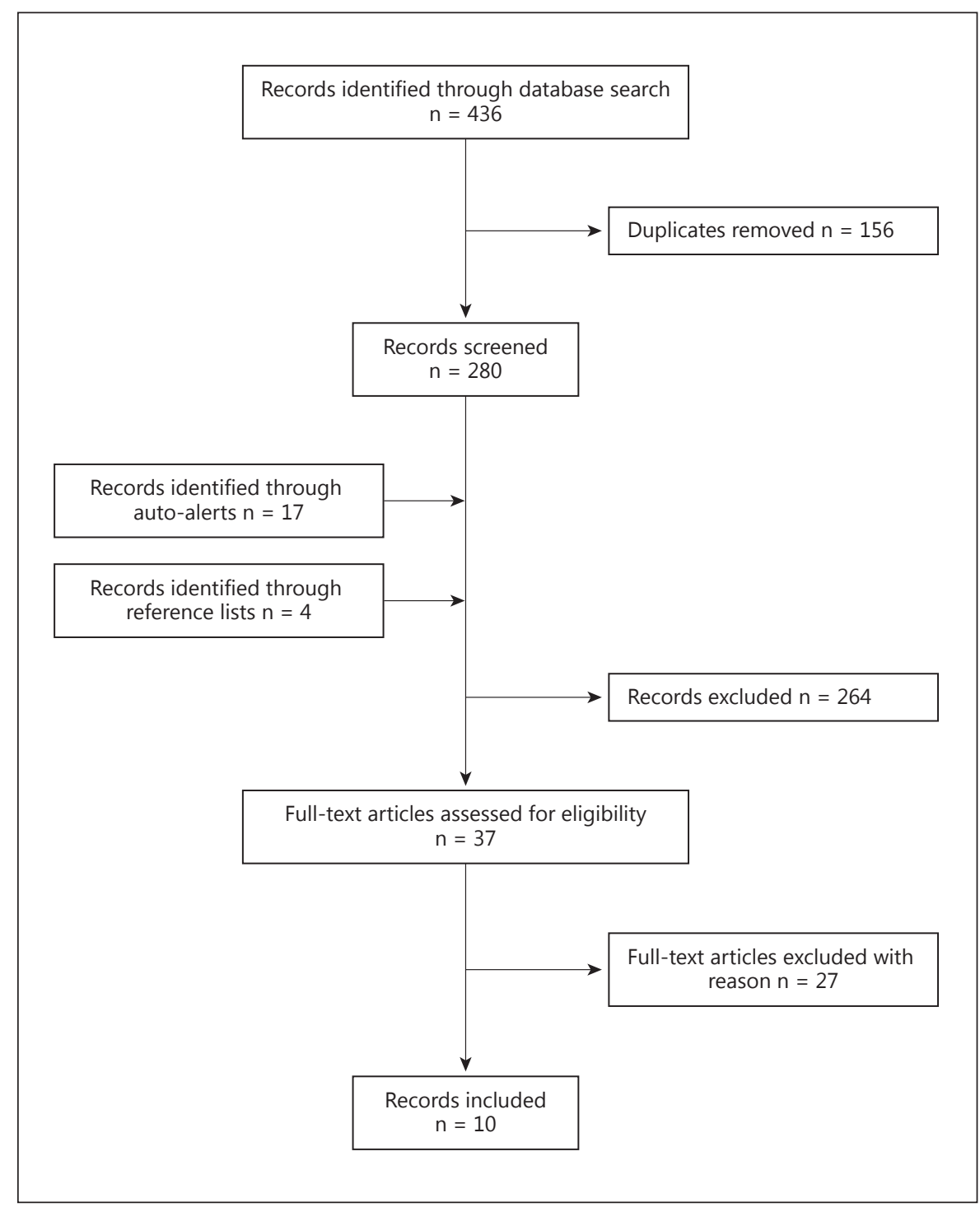

background, if stated, was: 100\% Mongoloid [6], 87.5\% Caucasian and $8.8 \%$ black [7], 75\% white and $15.5 \%$ black [8], $45 \%$ white and $36 \%$ black [9]. Each of the included maintenance RCTs was a follow-up of an initial RCT on induction therapy.

Therapies and reported outcome measures differed widely, hence pooling was only appropriate in 1 case. The timing of outcome assessment varied from 12 to 24 weeks. Results for each drug are reported in the next section.

\section{Adapalene Compared to Placebo}

Three placebo-controlled RCTs [6-8] evaluated adapalene $0.1 \%$ gel applied once daily (table 2). The RCTs included responders who had achieved 'at least moderate improvement' during the induction therapy phase (assessed using different scales in each study, see below).

In 2006, Thiboutot et al. [8] had conducted a 12-week induction RCT comparing adapalene $0.1 \%$ gel plus doxycycline $100 \mathrm{mg}$ compared to doxycycline $100 \mathrm{mg}$ alone. Responders, those who achieved at least moderate improvement, were then re-randomized to receive adapalene gel $0.1 \%$ q.d. or vehicle q.d. maintenance treatment for 16 weeks. The authors report a global severity assessment on a 6-point scale (from clear to worse) with $27 \%$ of participants still being 'clear/almost clear' in the adapalene arm and $16 \%$ of participants in the vehicle arm at the end of the maintenance phase. Statistically, no significant difference was found between the two groups 


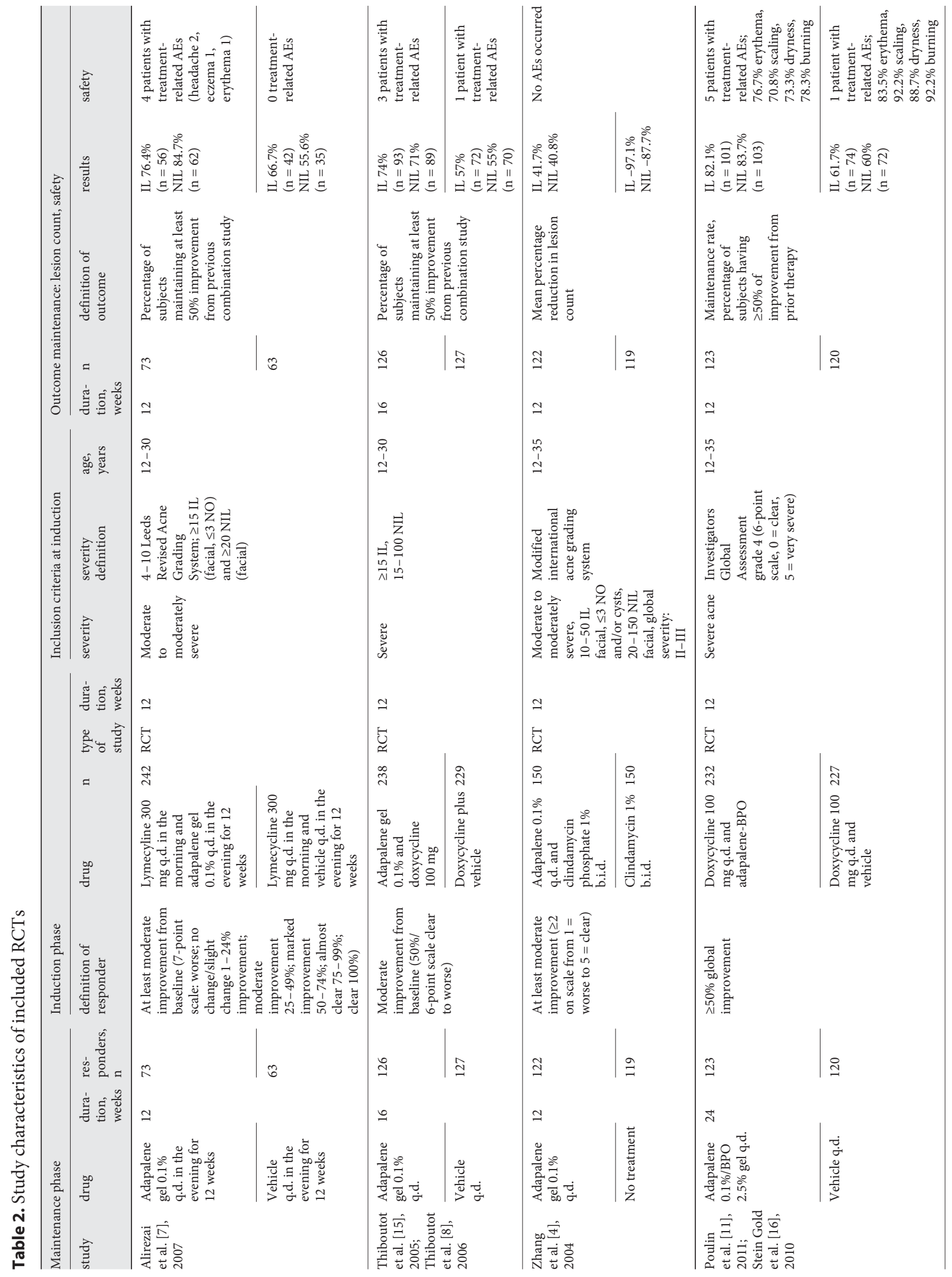




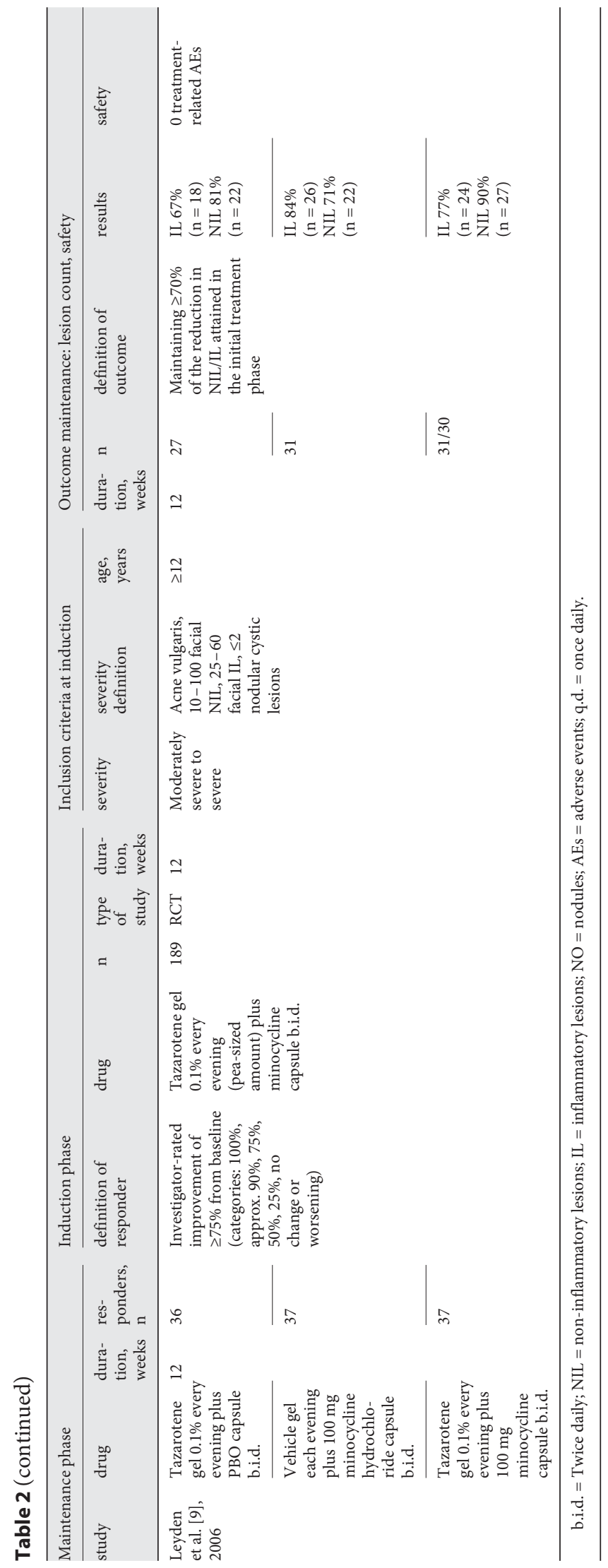

Maintenance Treatment of Acne with respect to the global severity assessment (risk ratio, RR 1.18; confidence interval, CI 0.77-1.82).

In 2007, Alirezei et al. [7] had initially compared adapalene gel $0.1 \%$ plus lymecycline $300 \mathrm{mg}$ to lymecycline $300 \mathrm{mg}$ plus vehicle. Responders, those who achieved at least moderate improvement after 12 weeks, entered a maintenance RCT whereby adapalene $0.1 \%$ gel q.d. was compared to vehicle gel q.d. Alirezei et al. stated that after 12 weeks of maintenance treatment, based on the Leeds Revised Acne Grading system of O'Brien et al. [10], the percentage of patients with 'mild acne' remained the same in the adapalene group (from 86.3 to $82.2 \%$ ) but decreased slightly in the vehicle group (from 76.2 to $68.3 \%$ ). No statistically significant difference could be found with regard to the grading system (RR 1.29, CI 0.99-1.47).

In contrast to the lack of statistical significance with respect to the grading used in the studies by Thiboutout et al. [8] and Alirezei et al. [7], the pooled effect of adapalene versus vehicle on the 'number of patients maintaining at least 50\% improvement achieved in the initial study' was statistically significant based on inflammatory (IL) and non-inflammatory lesion (NIL) count $[7,8]$ (fig. 3).

In 2004, Zhang et al. [6] had compared adapalene $0.1 \%$ gel plus clindamycin phosphate $1 \%$ versus clindamycin $1 \%$ during a 12 -week induction treatment RCT. Responders, those who achieved at least moderate improvement, were rerandomized to adapalene $0.1 \%$ gel q.d. or no treatment during the maintenance phase. Zhang et al. reported that $67.2 \%$ of patients in the adapalene group and $4.2 \%$ of patients in the placebo group reached 'global improvement' after 24 weeks (further improved or much improved $/$ clear on a scale from $-2=$ total relapse to $2=$ much improved/clear). A mean percentage reduction in total lesion count of $41.6 \%$ in the adapalene group and an increase of $92.1 \%$ in the placebo group were reported.

Adapalene plus BPO Compared with Vehicle

In 2010, Poulin et al. [11] had initially compared doxycycline plus BPO versus doxycycline monotherapy. Responders (those with $\geq 50 \%$ global improvement) were rerandomized to the fixed combination adapalene $0.1 \%$ / BPO $2.5 \%$ gel q.d. compared to vehicle q.d. as maintenance treatment. A statistically significant difference was found at 12 weeks based on IL count (RR 1.33, CI 1.131.57) and NIL count (RR 1.67, CI 1.38-2.04). This effect was larger after 24 weeks of maintenance treatment (IL count: RR 1.61, CI 1.31-1.99; NIL count: RR 1.80, CI $1.44-2.26)$. The authors also report that $70.70 \%$ of participants in the adapalene-BPO group had the same or a 
a IL count

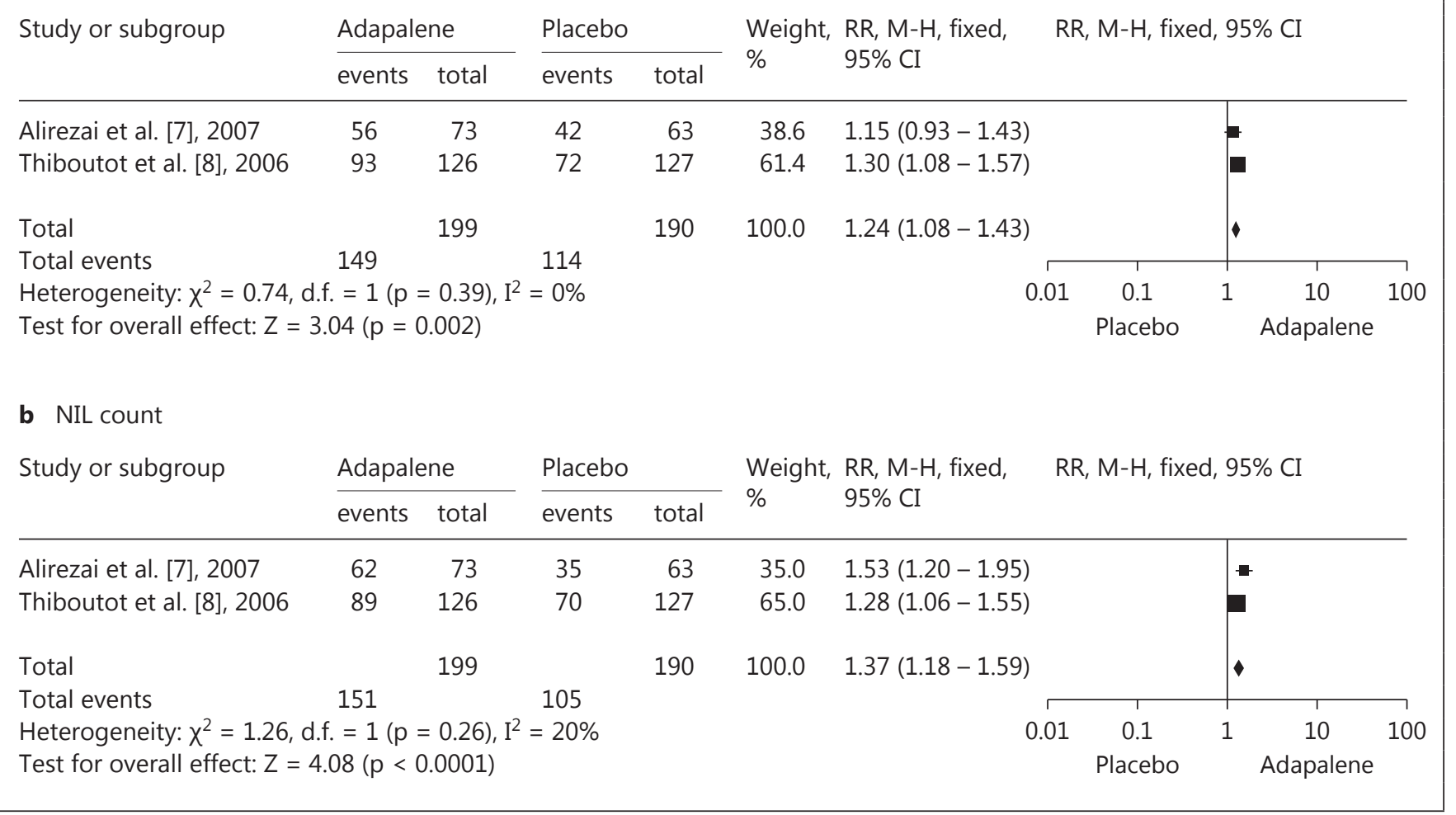

Fig. 3. Forest plot of pooled effect (risk ratio) of adapalene versus vehicle: number of patients maintaining $\geq 50 \%$ improvement based on IL (a) and NIL (b) counts. M-H = Mantel-Haenszel test.

better investigators' global assessment score (from $0=$ clear to $5=$ very severe) than at baseline maintenance compared to $34.20 \%$ of patients in the placebo group (RR 2.07 , CI 1.58-2.72). Relapse was reported for day 56 in the vehicle group and day 175 in the treatment group. It had been defined as: the time when less than 50\% improvement in $25 \%$ of participants or an investigators' global assessment score below baseline was achieved.

\section{Tazarotene plus Minocycline}

In 2006, Leyden et al. [9] conducted a maintenance RCT with patients who had successfully completed induction treatment with tazarotene $0.1 \%$ gel plus minocycline $100 \mathrm{mg}$ and achieved at least $75 \%$ global improvement. Patients were then randomized into three groups: tazarotene gel $0.1 \%$ every evening plus a placebo capsule twice a day (b.i.d.), or vehicle gel every evening plus minocycline hydrochloride $100 \mathrm{mg}$ capsules b.i.d., or tazarotene gel $0.1 \%$ every evening plus minocycline 100 mg capsules b.i.d.
No significant differences could be found with regard to the number of patients maintaining at least $70 \% \mathrm{im}$ provement comparing tazarotene alone with minocycline alone (based on IL count: RR 0.79, CI 0.58-1.08; NIL count: RR 1.15, CI 0.86-1.53), or tazarotene alone versus tazarotene plus minocycline (based on IL count: RR 0.86, CI 0.62-1.19; NIL count: RR 0.91, CI 0.73-1.12) or minocycline alone versus tazarotene plus minocycline (based on IL count: RR 1.08, CI 0.86-1.38; NIL count: RR 0.79, CI 0.61-1.02).

\section{Other Included Studies}

Three additional 12-week non-randomized studies were included (table 3 ). Note that all of these studies reported only descriptive results. The studies differed in previously used therapies and methods of re-assignment/ further treatment following the induction phase. These studies had recruited 261 participants. In 2013, Bettoli et al. [12] reported maintenance baseline data (mean age 21.2 years, range $13-38,47.83 \%$ women, $\mathrm{n}=70$ ). In 2011 , 


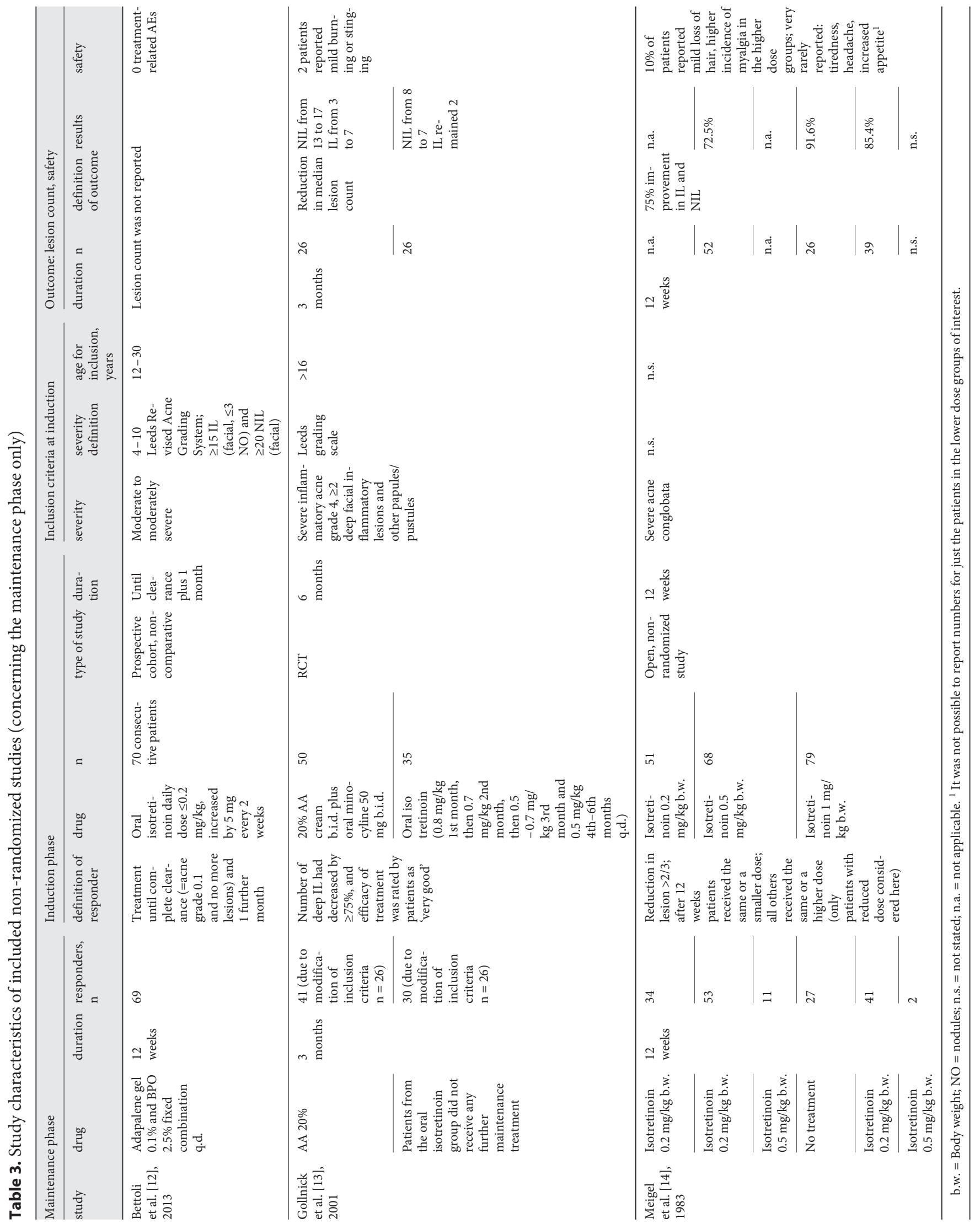




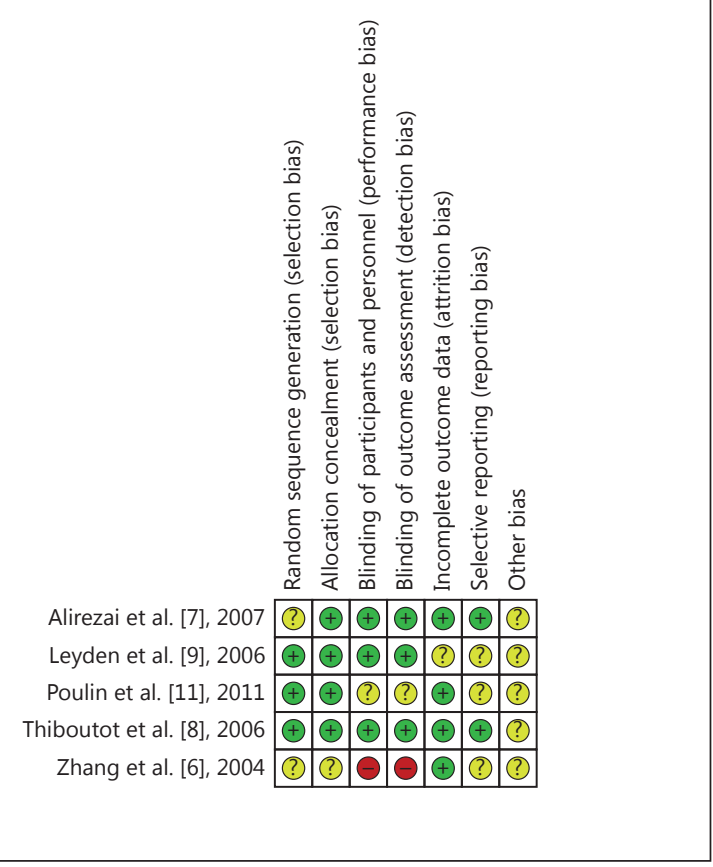

Fig. 4. Risk of bias of included RCTs.

Gollnick et al. [13] conducted a study with just men, and in 1983 Meigel et al. [14] provided no sociodemographics. Ethnic background or skin type were never reported.

\section{Adapalene plus BPO}

Bettoli et al. [12] conducted a non-randomized intervention study treating 69 responders (defined as complete clearance after treatment with oral isotretinoin $\leq 0.2 \mathrm{mg} / \mathrm{kg}$; duration specified as 'until clear') with adapalene $0.1 \% / \mathrm{BPO} 2.5 \%$ fixed combination q.d. The only reported outcome was relapse defined as the re-occurrence of acne severity grade $\geq 0.5$ ( 10 papules and pustules and 10 comedones), which was only experienced by 2 patients.

\section{Azelaic Acid}

Gollnick et al. [13] treated patients with AA 20\% who had been treated with AA $20 \%$ b.i.d. plus oral minocycline $50 \mathrm{mg}$ b.i.d. in the initial 6-month study and who had then achieved responder status (defined as number of deep inflammatory lesions that decreased by $\geq 75 \%$, and the improvement of treatment was rated by patients as 'very good'). The second original study arm received no maintenance treatment. After 12 weeks, the patients in the AA group experienced an increase in median lesion count (NIL from 13 to 17; IL from 3 to 7 ) whereas the notreatment group saw almost no change (NIL from 8 to 7 , IL remained 2). With regard to the global assessment of the patient's clinical appearance, in the AA group 38.5\% of participants were classified as showing marked deterioration and $15.4 \%$ as experiencing slight deterioration whereas in the no-treatment group 4 and $46.2 \%$ of participants were classified accordingly. Note that only a fraction of patients per group was evaluated.

\section{Isotretinoin}

Meigel et al. [14] evaluated isotretinoin $0.2 \mathrm{mg} / \mathrm{kg}$ body weight or no treatment in patients who had achieved at least a two-thirds reduction in lesions after 12 weeks of induction treatment with isotretinoin $0.2,0.5$ or $1 \mathrm{mg} / \mathrm{kg}$. The percentage of patients maintaining a $75 \%$ improvement in pooled NIL and IL lesion count was $72.5 \%$ in the patient group that was switched from 0.5 to $0.2 \mathrm{mg} / \mathrm{kg}$ $(\mathrm{n}=52), 91.6 \%$ in the $1 \mathrm{mg} / \mathrm{kg}$ switched to 'no-treatment' group $(\mathrm{n}=26)$ and $85.4 \%$ in the $1 \mathrm{mg} / \mathrm{kg}$ switched to 0.2 $\mathrm{mg} / \mathrm{kg}$ group $(\mathrm{n}=39)^{1}$

\section{Safety}

Drop-outs due to adverse events were rare (0-2 patients, reported in 6 studies). Tolerability was reported by all authors with mild but mostly no burning/stinging [13], erythema or dryness [6-9]. Further details are provided in table 2 .

\section{Risk of Bias}

Evaluations of the studies based on the seven predetermined risk of bias criteria are provided in figure 4 . The risk of other sources of bias was 'unclear' for all RCTs due to reasons such as authors associated with sponsor [8] or different/inconsistent/missing baseline data $[6,7,11]$. Attrition and performance bias were rated to have a low risk of bias in most RCTs (methods used for randomization and blinding described). Only Zhang et al. [6] failed to describe the former and conducted an open-label trial. The risk of outcome data being biased cannot be precluded.

The non-randomized studies were evaluated to have a moderate to serious risk of bias. In all three studies the risk of baseline confounder and the risk of selective re-

\footnotetext{
1 Note that only responders who received a lower dose (not the same dose) were considered for evaluation here because non-responders could also receive the same (or a higher) dose.
} 
porting were serious due to some missing baseline [14] or outcome data [12], or unknowable modification of inclusion criteria [13].

\section{Discussion}

In our systematic review we found 8 studies assessing acne maintenance treatment. All studies were conducted after patients had gone through induction therapy and achieved a predefined improvement response. However, initial treatments before the maintenance phase varied strongly and are very likely to influence the outcomes of the maintenance periods. It would be desirable to have study designs, in which patient populations receive an identical initial treatment and then responders are randomized into different maintenance regimens. In addition to the limitations in study design, the definitions of responders varied between the included studies making comparisons difficult.

The available evidence for adapalene as a maintenance treatment is somewhat conflicting, depending on the assessed outcome. A benefit of maintenance treatment with adapalene/BPO was seen. For tazarotene and minocycline alone or in combination, no relevant differences during maintenance treatment were seen.

Due to the evaluation of different outcome measures and the divergent baseline situations in the non-randomized intervention studies, no overall conclusions could be drawn from these.

\section{Limitations}

Our search was limited to two online databases, and no hand searches were conducted. The effect sizes were only reported for RCTs because these tend to be inflated in non-randomized studies. We pooled data across 12 and 16 weeks, which neglects the duration of treatment.

\section{Conclusion}

Pooled results somewhat support the use of topical treatments after the initial use of topical and systemic drugs.

The use of an appropriate maintenance study design (homogenous initial treatment phase study design, randomization into different maintenance regimens), standardized outcome measures and reporting of adverse events would be beneficial for the evaluation of different treatments.

\section{Statement of Ethics}

This is a systematic review, hence an IRB application was not required.

\section{Disclosure Statement}

This project was conducted as part of the update of the European Evidence-Based (S3) Guidelines for the Treatment of Acne funded by the European Dermatology Forum.

C.D. and S.R. declare no conflicts of interest. A.N. has received honoraria for educational activities with direct or indirect sponsoring by the following manufacturer of anti-acne treatments: Intendis/Bayer Healthcare (AA); A.N. has received honoraria for educational activities with direct or indirect sponsoring by the following companies, which, to the best of my knowledge, are no manufacturers of anti-acne treatments: Pfizer, Boehringer Ingelheim, Novartis, Biogen Idec, Abbott (now Abbvie).

The Division of Evidence-Based Medicine has received research grants from Pfizer for a systematic review on psoriasis maintenance therapy and a research grant from GlaxoSmithKline for a systematic review on time until onset of action of treatments for acne vulgaris. GlaxoSmithKline is a manufacturer of anti-acne treatments (BPO/clindamycin).

\section{References}

1 Poli F, Dreno B, Verschoore M: An epidemiological study of acne in female adults: results of a survey conducted in France. J Eur Acad Dermatol Venereol 2001;15:541-545.

2 Hayashi N, Miyachi Y, Kawashima M: Prevalence of scars and 'mini-scars', and their impact on quality of life in Japanese patients with acne. J Dermatol 2015;42:690-696.

-3 Moher D, Liberati A, Tetzlaff J, Altman DG: Preferred reporting items for systematic reviews and meta-analyses: the PRISMA statement. J Clin Epidemiol 2009;62:1006-1012.
4 Higgins JPT, Green S (eds): Cochrane Handbook for Systematic Reviews of Interventions. Oxford, Cochrane Collaboration, 2011.

5 Sterne J, Higgins J, Reeves B; development group for ACROBAT-NRSI: A Cochrane Risk of Bias Assessment Tool for Non-Randomized Studies of Interventions (ACROBAT-NRSI). 2014.

-6 Zhang JZ, Li LF, Tu YT, Zheng J: A successful maintenance approach in inflammatory acne with adapalene gel $0.1 \%$ after an initial treatment in combination with clindamycin topical solution $1 \%$ or after monotherapy with clindamycin topical solution $1 \%$. J Dermatolog Treat 2004;15:372-378. 
7 Alirezai M, George SA, Coutts I, Roseeuw DI, Hachem JP, Kerrouche N, et al: Daily treatment with adapalene gel $0.1 \%$ maintains initial improvement of acne vulgaris previously treated with oral lymecycline. Eur J Dermatol 2007;17:45-51.

8 Thiboutot DM, Shalita AR, Yamauchi PS, Dawson C, Kerrouche N, Arsonnaud S, et al: Adapalene gel, $0.1 \%$, as maintenance therapy for acne vulgaris: a randomized, controlled, investigator-blind follow-up of a recent combination study. Arch Dermatol 2006;142: 597-602.

-9 Leyden J, Thiboutot DM, Shalita AR, Webster G, Washenik K, Strober BE, et al: Comparison of tazarotene and minocycline maintenance therapies in acne vulgaris: a multicenter, double-blind, randomized, parallel-group study. Arch Dermatol 2006;142:605-612.
0 O’Brien S, Lewis J, Cunliffe W: The Leeds revised acne grading system. J Dermatolog Treat 1998;9:215-220.

-11 Poulin Y, Sanchez NP, Bucko A, Fowler J, Jarratt $\mathrm{M}$, Kempers S, et al: A 6-month maintenance therapy with adapalene-benzoyl peroxide gel prevents relapse and continuously improves efficacy among patients with severe acne vulgaris: results of a randomized controlled trial. Br J Dermatol 2011;164:13761382.

12 Bettoli V, Borghi A, Zauli S, Toni G, Ricci M, Giari S, et al: Maintenance therapy for acne vulgaris: efficacy of a 12-month treatment with adapalene-benzoyl peroxide after oral isotretinoin and a review of the literature. Dermatology 2013;227:97-102.

13 Gollnick HP, Graupe K, Zaumseil RP: Comparison of combined azelaic acid cream plus oral minocycline with oral isotretinoin in severe acne. Eur J Dermatol 2001;11:538-544.
14 Meigel W, Gollnick H, Wokalek H, Plewig G: Oral treatment of acne conglobata using 13-cis-retinoic acid. Results of the German multicentric study following 24 weeks of treatment (in German). Hautarzt 1983;34: 387-397.

15 Thiboutot DM, Shalita AR, Yamauchi PS, Dawson C, Arsonnaud S, Kang S: Combination therapy with adapalene gel $0.1 \%$ and doxycycline for severe acne vulgaris: a multicenter, investigator-blind, randomized, controlled study. Skinmed 2005;4:138-146.

16 Stein Gold L, Cruz A, Eichenfield L, Tan J, Jorizzo J, Kerrouche N, et al: Effective and safe combination therapy for severe acne vulgaris: a randomized, vehicle-controlled, doubleblind study of adapalene $0.1 \%$-benzoyl peroxide $2.5 \%$ fixed-dose combination gel with doxycycline hyclate $100 \mathrm{mg}$. Cutis 2010;85:94104. 